\title{
Two cases of Guillain-Barré syndrome after cerebral hemorrhage or head trauma
}

\author{
Huan Jia', Ye Tian', Yan-Min Wu${ }^{1}$, Bin $\mathbf{L i}^{1,2}$ \\ ${ }^{1}$ Department of Neurology, The Second Hospital of Hebei Medical University, Shijiazhuang 050000, Hebei, China. \\ ${ }^{2}$ Key Laboratory of Hebei Neurology, Shijiazhuang 050000, Hebei, China.
}

Correspondence to: Prof. Bin Li, Department of Neurology, The Second Hosipital of Hebei Medical University, Key Laboratory of Hebei Neurology, Heping West Road 215, Shijiazhuang 050000, Hebei, China. E-mail: jack511@163.com

How to cite this article: Jia H, Tian Y, Wu YM, Li B. Two cases of Guillain-Barré syndrome after cerebral hemorrhage or head trauma. Neuroimmunol Neuroinflammation 2017;4:61-4.

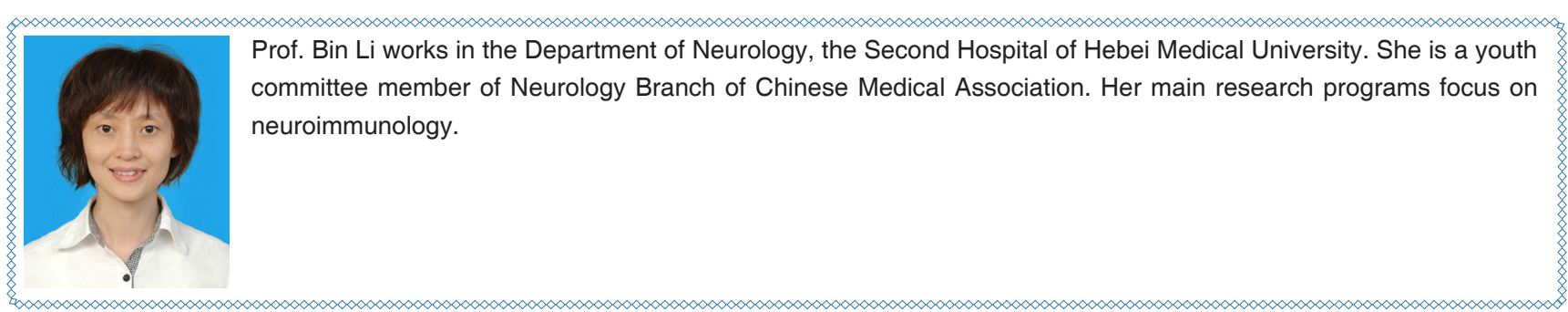

\section{Article history:}

Received: 23-11-2016

Accepted: 24-02-2017

Published: 12-04-2017

\section{Key words:}

Guillain-Barré syndrome,

cerebral hemorrhage,

trauma,

anti-ganglioside antibodies

\section{ABSTRACT}

Guillain-Barré syndrome (GBS) is an uncommon disease involving widespread peripheral nerve inflammatory demyelination which results in ascending symmetrical limb paralysis and areflexia. Approximately $2 / 3$ of cases occurred following a simple, trivial antecedent infection. In northern China, diarrhea caused by Campylobacter jejuni is the most common etiology of GBS. This article presents 2 cases - post cerebral hemorrhage and post head traumatic GBS. Both patients suffered from acute motor axonal neuropathy, a main subtype of GBS, 14 days after cerebral hemorrhage or head trauma without any antecedent infection. The possible pathophysiological mechanisms are discussed in the article, and the importance of increasing the awareness of early diagnosis, as well as early treatment with intravenous immunoglobulin and supportive care, in this special pathogenic GBS is emphasized.

\section{INTRODUCTION}

Guillain-Barré syndrome (GBS) is an acute inflammatory polyneuropathy that primarily damages human spinal nerve roots and peripheral nerves. One of the possible pathological changes is lymphocyte and macrophage infiltration around the small blood vessels of peripheral nerves, which causes demyelination. ${ }^{[1]}$ As far as we know, various factors can result in GBS. Almost $2 / 3$ of GBS patients suffer from bacterial or viral infections within 1-4 weeks before onset. Campylobacter jejuni (C. jejuni) is the most common pathogen of the prodromal infection especially in northern China. The most common pathogenesis of GBS is mainly 
based on hypothetical mechanisms deduced from molecular modeling of prodromal infections, caused by pathogens such as $C$. jejuni, which can activate the body's immune system to produce antibodies, resulting in peripheral nerve demyelination or axonal injury. In particular, serum anti-ganglioside antibodies in the acute phase are detectable in approxiately $60 \%$ of GBS patients. ${ }^{[2]}$

In recent years, weakness of extremities and weakened, or even loss of, tendon reflexes have been reported in some patients with traumatic brain injury, cerebral hemorrhage, spinal cord injury, brachial plexus injury, or pelvic fracture fixation..$^{[3-6]}$ In this article, we provide detailed clinical data of two patients who were diagnosed with GBS following brain hemorrhage or craniocerebral injury. The diagnosis of our 2 patients is based on the diagnostic criteria of GBS published in 2014, which was modified according to the criteria published in $1990 .{ }^{[7]}$

\section{CASE REPORT}

\section{Case 1}

A 33-year-old male suffered from right basal ganglia hemorrhage (moderate volume) and gradually regained his muscle strength (left arm $3 / 5$, left leg $2 / 5$, right limbs 5/5) (the Medical Research Council grading system) after receiving conservative treatment. The patient received conservative treatment immediately after brain hemorrhage including neurotrophic treatment, mannitol, antihypertensive treatment and so on, without any treatment with gangliosides injections, during which there was no evidence of infection such as fever, cough, or elevation of white blood cells. However, fourteen days later, the muscle strength of both lower limbs decreased sharply to $0 / 5$, the left arm $1 / 5$, and the right arm $2 / 5$, accompanied with hypomyotonia and the absence of tendon reflexes, without other pathological signs of the nervous system. A reexamined cranial computed tomography (CT) scan showed there were no new lesions, but his muscle weakness worsened rapidly. Besides, cranial magnetic resonance imaging (MRI) was performed on him, demonstrating moderate volume hemorrhage in the right basal ganglia region and no signs of new hemorrhage. MRI of the cervical vertebra showed mild disc (C2-C7) herniation. Unfortunately, his muscle strength continued to decrease, and five days later, all extremities decreased to $0 / 5$ and respiratory failure occurred. A nerve conduction study (NCS) indicated: compound muscle action potential (CMAP) amplitude of the right median nerve and the common peroneal nerve on both sides were not elicited; CMAP of the right ulnar nerve significantly decreased; $F$ wave was not evoked in both upper and lower limbs; and the motor nerve conduction velocity (NCV) was close to normal. The patient was transferred to the intensive care unit (ICU) right away, due to the respiratory problems, and a ventilator was used to keep him alive. We collected a cerebrospinal fluid (CSF) sample, and the analysis showed a protein concentration of $0.56 \mathrm{~g} / \mathrm{L}$, a normal cell count $\left(4 \times 10^{6} / \mathrm{L}\right)$ and the pressure of CSF was $170 \mathrm{mmH}_{2} \mathrm{O}$. The result of the serum antiganglioside antibody test was negative 17 days after the occurrence of muscle weakness. According to the diagnostic criteria, he was diagnosed with acute motor axonal neuropathy (AMAN), a subtype of GBS.

Intravenous immunoglobulin (IVlg) $0.4 \mathrm{~g} / \mathrm{kg}$ per day was given to him 5 days after the occurrence of quadriplegia for a consecutive 5 days of treatment. However, no significant improvement was observed. After 7 days, the same dose of gamma globulin was administered for another 5 days and rehabilitation treatment was continued. A month later, the ventilator was gradually discontinued and the patient regained some muscle strength (right arm 3/5, left arm $2 / 5$, and both legs $1 / 5$ ). Meanwhile the rehabilitation treatment continued. A 4-month follow-up revealed that he could walk slowly with some support by arm.

\section{Case 2}

A 41-year-old male with a left frontal contusion, laceration, and subdural hematoma, due to a motorcycle accident, was included into the case analysis. At first, the patient's conditions had improved obviously after conservative treatment. However, 2 weeks later, he suffered from sudden quadriplegia (muscle strength $0 / 5$ for all limbs) along with areflexia, without any evidence of antecedent infections and without treatments with gangliosides injections. Another brain CT scan was performed immediately, but no obvious changes were observed. Then the patient was transferred to the ICU because of respiratory failure, and a ventilator was used. NCS showed that: CMAP of the right median nerve and sural nerve were not elicited; CMAP of the common peroneal nerve on both sides were significantly decreased; $F$ waves were not evoked in either upper or lower limbs; NCV was close to normal. Lumbar puncture showed that the CSF pressure was $190 \mathrm{mmH}_{2} \mathrm{O}$, protein concentration was $1.87 \mathrm{~g} / \mathrm{L}$, and total nucleated cells were $2.0 \times 10^{6} / \mathrm{L}$. Diagnosis of GBS was definite and he was treated with IVIg $(0.4 \mathrm{~g} / \mathrm{kg}$ per day) for 5 days. During the treatment, his respiratory function recovered slightly, but with no improvement in muscle strength in any limb. Over another 5-day course of IVIg, his muscle strength recovered to $2 / 5$ in both arms but remained $0 / 5$ in the lower limbs. Six months later, the patient could gradually stand up with 
some support and hold light objects.

\section{DISCUSSION}

We reported two patients with severe GBS following cerebral hemorrhage or head trauma. In the two cases above, typical clinical manifestations were the sudden occurrence of flaccid tetraplegia, areflexia, hypomyotonia, and respiratory failure, without any sensory dysfunction. NCS of both patients showed decreased CMAP and prolonged distal $F$ wave latencies, indicating motor axon injury, which are common electrophysiological features of AMAN. In addition, the result of the CSF tests indicated albumino-cytologic dissociation, and IVIg proved to be efficient in both patients.

The most common subtypes of GBS are acute inflammatory demyelinating polyradiculoneuropathy (AIDP) and AMAN. In contrast to North America and Europe, where more than $90 \%$ of the patients are classified as AIDP, approximately $65 \%$ of patients in China suffer from AMAN, a subtype of GBS with only motor fiber damage..$^{[8]}$ Analogous to our cases, the features of nerve conduction studies demonstrated decreased motor amplitudes with an absence of $F$ waves.

The exact pathogenesis of GBS following brain trauma and hemorrhage is not completely clear. Tan et al. ${ }^{[6]}$ performed sural nerve biopsy and found phagocytosis of myelin sheath debris in the endoneurium. Tan suggested that head trauma and surgery had elevated serum and CSF myelin basic protein levels, which led to immune system activation to produce antimyelin antibodies that cause demyelination. Moreover, relevant literature indicates that blood-brain barrier (BBB) damage plays an important role in posttraumatic GBS pathogenesis. ${ }^{[9,10]}$ Disintegration of the BBB during neurotrauma leads to the accumulation of localized T lymphocytes and macrophages, which may induce the transformation of microglial cells in the nervous system into antigen presenting cells. ${ }^{[3,11,12]}$ Activated microglia can present post traumatic neuronal debris to the immune system and stimulate B cells to produce antibodies against the myelin sheath, causing demyelination, especially in the peripheral nervous system. ${ }^{[13]}$ The explanation of GBS following head trauma or hemorrhage may be that some substances originating in the central nervous system, usually being weakly immunogenic, are transported through the disrupted BBB to the peripheral nervous system where they cause demyelination or axonal damage.

It has been reported that anti-ganglioside antibodies are elevated in patients with post traumatic head injury. ${ }^{[3]}$ Some studies have shown that nearly $80 \%$ of AMAN patients are anti-ganglioside antibody positive, especially within the first week of onset. ${ }^{[4]}$ The best treatment for GBS is IVIg or plasma exchange, both of which aim to remove antibodies. However, serum anti-ganglioside antibodies were negative in case 1 . We postulate 2 possible explanations: one is that the antibody level had decreased sharply after 2 courses of IVIg when it was obtained 17 days after onset; the other is the patient suffered from anti-ganglioside antibody negative GBS. The 2 patients reported showed significant recovery due to prompt diagnosis and treatment.

In clinical practice, if a sudden bilateral limb weakness occurs after traumatic injury or hemorrhage, which cannot be explained by findings of imaging and routine laboratory examinations, GBS should be considered. ${ }^{[14]}$ Early examination of the nervous system, EMG, CSF test, and search for anti-ganglioside antibodies can support the diagnosis of peripheral nerve demyelinating disease. For patients diagnosed with GBS, gamma globulin should be given as soon as possible, if there are no contraindications, otherwise plasma exchange can be used. ${ }^{[15]}$ Despite the rare reports of GBS following head trauma or brain hemorrhage, we find that it is not as rare as we thought in clinical practice. GBS is not a common disease and it is easy to be ignored or misdiagnosed, especially following other severe situations like trauma and brain hemorrhage. Thus, it is of great significance to enhance the awareness of early diagnosis and early treatment of this special pathogenic GBS, which will increase the survival rate and improve the quality of patients' life.

\section{Authors' contributions}

Collection of study cases and problem analysis: $\mathrm{H}$. Jia Writing of the paper and critical revision of the article: H. Jia, B. Li

Revised article: H. Jia, Y. Tian, Y.M. Wu

\section{Financial support and sponsorship}

None.

\section{Conflicts of interest}

There are no conflicts of interest.

\section{Patient consent \\ Consent was approved by patients in both cases.}

\section{Ethics approval}

Data collection in our study involving the patient is consistent with the ethical standards of the institution's ethics committee. 


\section{REFERENCES}

1. Yuki N, Hartung HP. Guillain-Barré syndrome. $J$ Med 2012;366:2294-304.

2. Wu XK, Cong ZQ, Deng H. Research progress about anti-ganglioside in Guillain-Barré syndrome. J Apoplexy Nerv Dis 2013;30:1133-5.

3. Carr KR, Shah M, Garvin R, Shakir A, Jackson C. Post-Traumatic brain injury presenting with Guillain-Barré syndrome and elevalted anti-ganglioside antibodies: a case report and review of the literature. Int J Neurosci 2015;125:486-92.

4. Rivas S, Douds GL, Ostdahl RH, Harbaugh KS. Fulminant GuillainBarré syndrome after closed head injury: a potentially reversible cause of an ominous examination. J Neurosurg 2008;108:595-600.

5. Wu Q, Liu N, Pan C, Bu B, Tang Z. Guillain-Barré syndrome and cerebral hemorrhage: two cases and literature review. Eur Neurol 2016;76:182-6.

6. Tan IL, Ng T, Vucic S. Sever Guillain-Barré syndrome following head trauma. J Clin Neurosci 2010;17:1452-4.

7. Van den Berg B, Walgaard C, Drenthen J, Fokke C, Jacobs BC, Van Dorrn PA. Guillain-Barré syndrome: pathogenesis, diagnosis, treatment and prognosis. Nat Rev Neuro 2014;10:469-82.

8. Wang YC, Feng GD, Wang J, Liu XD, Zhao G. Effect of conduction block in classification and prognosis of Guillain-Barre syndrome. Neuroimmunol Neuroinflammation 2014;1:77-81.

9. Kanda T. Biology of the blood-nerve barrier and its alteration in immune mediated neuropathies. J Neurol Neurosurg Psychiatry 2013;84:208-12.

10. Kanda T. Yamawaki M, Mizusawa H. Sera from Guillain-Barré patients enhance leakage in blood-nerve barrier model. Neurology 2003;60:301-6.

11. Morganti-Kossmann MC, Satgunaseelan L, Bye N, Kossmann T. Modulation of immune response by head injury. Injury 2007;38:1392-400.

12. Shrikant P, Benveniste EN. The central nervous system as an immunocompetent organ: role of glial cells in antigen presentation. $J$ Immunol 1996;157:1819-22.

13. Kreutzberg GW. Microglia: a sensor for pathological events in the CNS. Trends Neurosci 1996;19:312-8.

14. Willison HJ, Yuki N. Peripheral neuropathies and antiglycolipid antibodies. Brain 2002;125:2591-625.

15. Mohr M, Englisch L, Roth A, Burchardi H, Zielmann S. Effects of early treatment with immunoglobulin on critical illness polyneuropaththy following multiple organ failure and Gram-negative sepsis. Intensive Care Med 1997;23:1144-9. 